\title{
Transplante de órgãos pélvicos: uma nova proposta de tratamento para incontinência fecal e urinária
}

\author{
Pelvic organ transplantation: a new proposal for treatment \\ of fecal and urinary incontinence
}

\begin{abstract}
Leandro Ryuchi Iuamoto ${ }^{1}$, Maria Clara de Camargo Traldi², Victor Subyung Lee ${ }^{3}$, Jun Araki ${ }^{4}$, Flávio Henrique Ferreira Galvão ${ }^{5}$, Luiz Augusto Carneiro D’Albuquerque ${ }^{6}$
\end{abstract}

Iuamoto LR, Traldi MCC, Lee VS, Araki J, Galvão FHF, D’Albuquerque LAC. Transplante de órgãos pélvicos: uma nova proposta de tratamento para incontinência fecal e urinária / Pelvic organ transplantation: a new proposal for treatment of fecal and urinary incontinence. Rev Med (São Paulo). 2015 jan.-mar.;94(1):36-45.

RESUMO: Incontinência fecal e urinária é um problema atual de saúde pública, com enorme impacto social e econômico. Tratamentos para esse problema são insuficientes. Após investigação anatômica, percebemos o assoalho pélvico como tecido composto independente que compartilha pedículo vascular (ilíaca interna) e neuronal (pudendo) comum. Neste manuscrito nós descrevemos transplante de órgãos pélvicos em ratos e cadáveres. Doador - Uma incisão combinada perineal e abdominal foi realizada. A dissecção progrediu perto do anel pélvico; o nervo pudendo e seus vasos foram divididos permitindo a mobilização total do assoalho pélvico. Através da incisão abdominal, genitália, bexiga e reto foram mobilizados. A aorta e a veia cava foram isoladas, preservando os vasos ilíacos internos e os vasos espermáticos. Os enxertos contendo pele, complexo muscular, ligamentos, bexiga, uretra, vagina ou pênis, reto, ânus, útero, ovário, testículo e seus condutos foram removidos. Receptor - A mesma estratégia de incisão e dissecção do doador foi realizada. Os vasos ilíacos internos, incluindo vasos retais e nervos pudendo foram divididos e os tecidos do assoalho pélvico foram removidos em bloco. A aorta e a veia cava infra-renal foram isolados, o enxerto foi colocado no abdômen e foi realizada anastomose aorta-aorta e cava-cava. Também realizamos anastomoses do ureter, reto e nervos e vasos pudendo. $\mathrm{O}$ assoalho pélvico foi colocado em sua posição original por pontos entre os ligamentos do assoalho pélvico, nervos e vasos pudendo, peritônio e da pele, concluindo a operação. Nesta pesquisa, mostramos que a cirurgia de transplante do assoalho pélvico é viável.

DESCRITORES: Incontinência fecal; Incontinência urinária; Transplante de tecidos; Canal anal; Genitália; Ovário; Útero; Literatura de revisão como assunto.

$1^{\circ}$ lugar Prêmio Monografias no 33 COMU - Congresso Médico Universitário da FMUSP, SP, 31 de out. a 02 de nov. de 2014.

Trabalho desenvolvido na Faculdade de Medicina da Universidade de São Paulo (FMUSP).

1. Acadêmico do Curso de Medicina da Faculdade de Medicina da Universidade de São Paulo. E-mail: leandro.iuamoto@gmail.com.

2. Acadêmica do Curso de Medicina da Faculdade de Ciências Médicas da Santa Casa de São Paulo. E-mail: mariaclaratraldi@yahoo.com.br.

3. Acadêmico do Curso de Medicina da Faculdade de Ciências Médicas da Santa Casa de São Paulo. E-mail: victorlee00131@gmail.com.

4. Researcher, Surgeon and PhD student at Department of Plastic Surgery, University of Tokyo. E-mail: ja980079@yahoo.co.jp/arakij-pla@ hu.tokyo.ac.jp.

5. Professor Livre-Docente, Disciplina de Transplante e Cirurgia do Fígado da Faculdade de Medicina da Universidade de São Paulo. E-mail: fgalvao@usp.br.

6. Professor Titular da Disciplina de Transplante de Órgãos do Aparelho Digestivo do Departamento de Gastroenterologia da Faculdade de Medicina da Universidade de São Paulo. E-mail: profluizcarneiro@gmail.com.

Endereço para correspondência: Avenida Doutor Arnaldo, 455 - Sala 3206. CEP: 01246-903 - Cerqueira César, São Paulo, SP, Brasil. E-mail: leandro.iuamoto@gmail.com. 
ABSTRACT: Fecal and urinary incontinence (FUI) is a current public health problem with enormous social and economic impact. Treatments for FUI are insufficient. After anatomic investigation we perceived pelvic floor as an independent block composite tissue sharing a common vascular pedicle (external iliac) and neuronal trunk (pudenda). In this report, we describe pelvic organs transplantation in cadavers and rats. Donor operation - A combined perineal and abdominal incision was performed. The dissection progress near the pelvic ring and pudendal nerves and vessels were divided allowing the total pelvic floor mobilization. Through the abdominal incision, the genitalia, bladder and rectum were mobilized. The aorta and vena cava were isolated preserving the internal iliac vessels and the spermatic vessels. The graft containing skin, muscular complex, ligaments, bladder, ureter, vagina or penile, rectum, anus, uterus, ovarian, testis and its ducts

\section{INTRODUÇÃO}

A incontinência fecal e urinária, também chamada de incontinência dupla (ID) é causada pela disfunção do assoalho pélvico e provoca grave problema na saúde pública mundial (1-8). Sujar as vestes, eliminar flatus sem controle e a perda inadvertida de fezes e urina são condições incapacitantes que tem impacto altamente negativo na qualidade de vida. Pacientes com ID grave frequentemente levam vida de desespero silencioso e isolamento social ${ }^{1-7}$.

A prevalência de incontinência urinária em adultos de meia-idade, citada na literatura médica, situa-se entre $14 \% \mathrm{e}$ $41 \%$ e a prevalência da incontinência anal em mulheres com sintomas de incontinência urinária é estimada entre $20 \%$ e $30 \%{ }^{2-5}$. A incidência da incontinência fecal situa-se entre 2,3 a $24 \%$ da população de adultos e essa taxa aumenta com idade $^{5}$. Aproximadamente $40 \%$ dos homens e mulheres com incontinência anal também têm incontinence urinária ${ }^{6}$. Isso se deve ao fato desses esfincteres compartilharem grupos musculares e mesmo pedículo vasculo-nervoso (artérias e veias iliaca interna, e pudendo) $)^{1-7}$. Pacientes com ID têm deficiência física e psicossocial significativamente maior do que os que possuem apenas incontinência fecal ou urinária isoladamente ${ }^{8}$.

A ID também representa significativo fardo econômico para a sociedade e para os sistemas de saúde. Estima-se que o impacto econômico anual varie de 16 a 26 bilhões de dólares ${ }^{9-11}$. O tratamento da ID é complexo e multidisciplinar, com resultados pouco efetivos, sobretudo nas disfunções graves do assoalho pélvico ${ }^{1-11}$. Neste manuscrito revisaremos o impacto da ID e discutiremos o tratamento desse problema, com ênfase a novas propostas terapêuticas, incluindo o transplante de órgãos pélvicos.

\section{MÉTODO}

Para a redação deste manuscrito utilizamos as bases de dados Cochrane Library, Medline e Embase e como palavras-chave os seguintes descritores: urinary incontinence AND fecal incontinence AND ("1990"[Date was removed. Recipient operation - The same donor's incision and dissection strategies were performed. The internal iliac vessels including rectal vessels and pudendal nerves were divided and the pelvic floor block tissues are removed. The infrarenal aorta and vena cava were isolated, the graft was positioned in the abdomen and an aorta-aorta such as a cava-cava anastomosis were performed. We also performed anastomosis between donor and recipient ureter, rectum and pudenda nerves and vessels. The pelvic floor was repositioned in its original position by stitches between pelvic floor ligaments, pudendal nerves and vessels, peritoneum and skin, completing the operation. In this survey, we show that pelvic floor transplantation surgery is feasible.

KEYWORDS: Fecal incontinence; Urinary incontinence; Tissue transplantation; Anal canal; Genitalia; Ovary; Uterus; Review literature as topic.

- Completion]: “3000”[Date - Completion], obtendo-se 2229 artigos.

Como critério de elegibilidade dos artigos, avaliamos os tipos de estudos (trabalhos randomizados controlados, não randomizados controlados, estudos observacionais), os tipos de participantes (estudos clínicos em adultos maiores de 18 anos), e o período de tempo de publicação (entre 1990 e 2014). E como critério de exclusão, artigos com dados sem relevância para o tema dessa monografia. Assim, foram revisados 379 artigos relacionados ao tema, e utilizados 62 , que apresentavam relação objetiva com o presente artigo.

Os artigos selecionados são discutidos nesta revisão de acordo com a seguinte divisão: função e anatomia do assoalho pélvico, tipos de incontinência fecal e urinária, etiologia, complicações da ID e tratamento, com ênfase no transplante de órgãos pélvicos.

\section{FUNÇÃO E ANATOMIA DO ASSOALHO PÉLVICO}

O assoalho pélvico possui duas funções inerentemente conflitantes: uma é fechar as cavidades pélvica e abdominal, de modo a suportar a carga dos órgãos viscerais; a outra é controlar as aberturas do reto e órgãos urogenitais que perfuram o assoalho pélvico. Para atingir ambas as tarefas, o assoalho pélvico é composto de várias folhas sobrepostas de músculos e tecidos conjuntivos, chamados de diafragma pelvico ${ }^{12}$.

O diafragma pélvico é composto do grupo Elevador Anal e inclui os músculos pubococcígeo, puboretal e o ileococcígeo do levantador do ânus e o músculo coccígeo. Eles surgem entre a sínfise púbica e a espinha isquiática, e convergem no cóccix e no ligamento anococcígeo, o qual se estende entre a ponta do cóccix e o hiato anal. Isto deixa um espaço para as aberturas anal e urogenital. O diafragma urogenital é constituído principalmente do músculo transverso profundo do períneo, que surge 
do ramo isquiático e púbico inferior e se estende até o hiato urogential. O diafragma urogenital é reforçada posteriormente pelo músculo transverso superficial do períneo ${ }^{13}$.

Os esfíncteres anais e uretral externo fecham o ânus e a uretra, estão em continuidade com estes músculos e ambos recebem inervação do pudendo. O primeiro é cercado pelo músculo bulboesponjoso, o qual restringe o intróito vaginal em mulheres e envolve o corpo esponjoso no sexo masculino; e pelo músculo isquiocavernoso, que obstrui a passagem do sangue para o corpo cavernoso do pênis e clitoris ${ }^{13}$.

\section{TIPOS E GRAUS DE INCONTINÊNCIA FECAL E URINÁRIA}

O grau de incontinência fecal é classicamente descrito pelo score de Williams ${ }^{12}$, dividido em cinco graus, de acordo com a presença de incontinência para gases, para fezes líquidas e para fezes sólidas (grau I, incontinência ocasional para gases; grau II, incontinência frequente para gases; grau III, incontinência ocasional para fezes líquidas; grau IV, incontinência ocasional para fezes sólidas; grau $\mathrm{V}$, incontinência frequente para fezes sólidas). Estas escalas, embora úteis pela simplicidade do estadiamento, não incluem a frequência das queixas ao longo do dia ou da semana.

Tem maior validade as escalas em que se avalia a frequência e também o impacto na qualidade de vida. Uma das mais utilizadas é a de Wexner ${ }^{14}$, num score de 0 a 20, que se baseia na frequência de episódios de incontinência para gases, fezes líquidas ou sólidas, bem como na necessidade de utilizar penso anal e na alteração da qualidade de vida, em que cada um deste cinco critérios são graduados de 0 a 4 (1, raramente; 2, às vezes; 3 , semanalmente; 4, diariamente).

As três formas principais de incontinência urinária são tensão, urgência e transbordamento ${ }^{1}$. A incontinência de tensão representa de 50 a $70 \%$ dos casos, que acontece quando a pressão intra-abdominal é elevada acima da resistência uretral. Isto pode acontecer enquanto a pessoa tosse ou espirra, curva-se, ergue um objeto pesado ou participa de atividades atléticas. Outros 20-40\% são incontinência de urgência, que é a inabilidade de inibir vazamento de urina por tempo suficiente para alcançar o banheiro quando a pessoa sente o desejo de urinar. Só $5-10 \%$ são casos de incontinência de transbordamento que acontece quando a bexiga não pode esvaziar completamente por causa de obstruções ou perda de força no músculo da bexiga, e assim, esta se torna flácida. Isto conduz a frequente, e às vezes quase constante, perda de urina ${ }^{1}$.

\section{ETIOLOGIA}

A incontinência fecal e urinária possui causas diversas envolvendo tanto homens quanto mulheres de todas as idades, raças e níveis socioeconômicos. Podem também estar associados a circunstâncias, como distúrbio hormonal, parto (o trabalho de parto pode resultar em estiramento e a degeneração parcial do nervo pudendo), cirurgia prévia, deficiência ou comprometimento muscular e traumas ${ }^{15,21}$. É importante notar que a incontinência fecal em homens está intimamente ligada ao histórico prévio de cirurgias anorretais ${ }^{16}$.

Muitos desses fatores estão interligados e não estão necessariamente relacionados ao envelhecimento. Geralmente estão associados à causa primária da IF, já que tal processo pode culminar na redução da força de contração do músculo detrusor, bem como na diminuição da capacidade vesical e na habilidade de contenção urinaria. Além disso, a prevalência de IF após cirurgia de ressecção retal por câncer é alta, já que o procedimento cirúrgico inclui remoção do reservatório retal com frequente dano do sistema de excreção urinária ${ }^{18}$. O processo de ressecção do colón e eventual radioterapia pode favorecer a ocorrência de diarreias e, portanto, perda fecal. Neste caso, a diarreia crônica se configura como fator predisponente de IF em ambos os sexos. A diarreia isoladamente muitas vezes não conduz a IF, porém o surgimento desta disfunção pode ser facilitado quando concomitante a outras mudanças nos mecanismos de controle da continência fecal.

Cirurgias de hemorroida também podem ser incluídas como causa da IF, pois nesse procedimento pode haver possível dano do esfíncter anal interno e perda dos mecanismos de contenção fecal ${ }^{19}$. Além disso, a presença de fístulas ou fissuras anais, bem como as cirurgias para correção desses problemas também são causas de $\mathrm{IF}^{20}$.

Dentre as causas mais comuns de incontinência urinária estão: infecções vaginais, constipação ou mal funcionamento do trato gastrointestinal, parto vaginal, fraqueza dos músculos que envolve a uretra e sustentam a bexiga, obstrução da uretra pelo aumento do volume da próstata (câncer), doenças que acometem o sistema nervoso ou muscular (derrame cerebral, esclerose múltipla, poliomielite, distrofia muscular, etc.) e alguns tipos de cirurgia ginecológicas ${ }^{21}$.

Os nervos que normalmente controlam a bexiga podem ser responsáveis pela hiperatividade vesical. Pacientes com doenças neurológicas frequentemente apresentam incontinência urinária como resultado da perda do controle normal sobre os nervos da bexiga ${ }^{15}$.

A maioria das obstruções é causada pelo aumento do volume da próstata. Desta maneira são geralmente associados aos casos de câncer ou cirurgia prostática, sendo, portanto, mais comuns nos homens ${ }^{23}$. 


\section{COMPLICAÇÕES}

$\mathrm{O}$ custo econômico associado à disfunção de assoalho pélvico em mulheres é substancial, nos Estados Unidos $^{24}$. Estimou-se que o custo direto anual apenas associado ao cuidado ambulatorial das disfunções do assoalho pélvico (PFDs), entre 1996 a 1997, foi de 190 milhões de dólares. Entre 2005 e 2006, de 298 milhões de dólares. Levando-se em considerações os custos indiretos, ou seja, aquelas não cobertos pelos planos de saúde, esse valor aumenta para 412 milhões de dólares.

Esse valor vem aumentando recentemente, o que pode ser resultado do aumento da prevalência das PFDs, devido ao envelhecimento da população. Também é importante ressaltar que a incontinência dupla é condição crônica, sem proposta de tratamento cirúrgico, de maneira que requerem um tratamento clínico, resultando, assim, em gastos continuados. No Brasil, não há dados sobre os custos com tratamento, e o número de PFDs é subestimado, porém se estima que a incidência acompanhe a dos países com mesmo nível de desenvolvimento e expectativa de vida, como os EUA.

É sabido que a incontinência urinária (IU) está associada a inúmeras comorbidades. Entretanto, ainda não é claro se ela e a comorbidade têm a mesma etiologia. Assim, não se sabe se a relação entre elas é causal, ou se o tratamento de uma condição exacerba ou melhora a outra ${ }^{30}$.

As principais comorbidades associadas às incontinências são:

\section{- Quedas e fraturas}

O risco de queda é uma preocupação principalmente em pacientes idosos com incontinência, provavelmente devido à pressa para chegar ao banheiro antes que o vazamento ocorra ${ }^{25}$. Como esses pacientes são mais sujeitos a fratura e, em geral, tem mobilidade debilitada, eles são mais suscetíveis a quedas e fraturas.

Segundo os dados da Leicestershire MRC Incontinency Study ${ }^{25}$, em um grupo de indivíduos maiores de 70 anos de idade, $25 \%$ apresentaram IU, e $35 \%$ reportaram quedas. A taxa de IU era maior nos indivíduos que reportaram quedas $(34,5 \%)$, do que naqueles que não apresentaram quedas.

\section{- Infecções do trato urinário}

Existem evidências que apontam para a associação entre infecções do trato urinário com incontinência urinaria, apesar das causas não serem conhecidas. O estudo NOBLE, mostra maior prevalência de incontinência urinária (IU) em mulheres com infecção urinária diagnosticada, comparada a mulheres sem $\mathrm{IU}^{26}$.

\section{- Obesidade}

Existe evidência que permite a associação entre o índice de massa corpórea (IMC) e obesidade com os diferentes tipos de incontinência urinária, ainda são necessários estudos para identificar as causas dessa associação. No estudo Saúde e Aposentadoria (HRS), realizado nos Estados Unidos com 8581 mulheres com mais de 50 anos de idade, mostrou que um dos preditores importantes de incontinência urinaria de urgência era a obesidade $^{27}$.

Já no estudo realizado em Boston, mostra que, dentre as 2403 mulheres sintomáticas, havia prevalência de IU de urgência (52\%) entre mulheres com altos IMC $\left(30-35 \mathrm{~kg} / \mathrm{m}^{2}\right)^{28}$.

\section{- Ansiedade e Depressão}

Os dados de Leicestershire MRC Incontinency Study, com 12.568 mulheres com idade maior de 40 anos no Reino Unido, indicou que incontinência urinária de urgência foi um fator de risco significativo para a ansiedade e depressão ${ }^{29}$. Supõe-se que as vias serotoninérgicas e noradrenérgicas sejam a ligação entre essas patologias, já que ambas estão envolvidas na micção e na ansiedade/ depressão. Porém, essa associação ainda não é clara e precisa de mais estudos ${ }^{30}$.

As comorbidades e o alto custo de tratamento mostram como a incontinência é um fardo para os pacientes, tendo um impacto negativo em suas vidas.

\section{TRATAMENTO}

A alta complexidade do mecanismo fisiológico de continência fecal e urinária faz com que o tratamento das disfunções que causam a ID seja extremamente difícil ${ }^{1}$. As opções terapêuticas para a ID têm mostrado resultados a longo prazo pouco animadores, especialmente em casos mais graves, o que abre espaço para o surgimento de novas opções de tratamento ${ }^{31}$.

\section{- Tratamento medicamentoso}

$\mathrm{O}$ uso de tratamento medicamentoso para incontinência fecal, com uso de formadores de massa, anticolinérgicos, opióides, antidepressivos, e outras drogas, são eficazes apenas na minoria dos casos de incontinência leve ou como medidas adicionais a outros tipos de tratamento ${ }^{32}$. Poucos estudos controlados mostraram melhoria de pacientes com incontinência urinária de tensão com o uso de medicamentos, estrógenos, agentes de estimulação etc. ${ }^{27}$. A incontinência urinária de transbordamento e urgência são melhoradas, às vezes, por meio de manipulações farmacológicas. Drogas anticolinérgicas são potentes inibidoras de contrações e os agentes de bloqueio podem ajudar quando a incontinência urinária de transbordamento é secundária à obstrução do pescoço da bexiga, como no caso da hipertrofia da próstata $^{27}$.

Outras medidas terapêuticas que surtem algum efeito para casos menos graves são o uso plugs e o biofeedback, sendo este último muito aplicado também 
como medida complementar ao tratamento cirúrgico ${ }^{33,34}$.

Dessa forma, analisamos os atuais métodos direcionados à recuperação de casos mais graves de incontinência nos itens: esfincteroplastia; graciloplastia; esfíncter artificial; neuroestimulação sacral; e, por último, um promissor método de tratamento - o transplante anorretal.

\section{- Esfincteroplastia}

A esfincteroplastia (ou reparação do esfíncter anal) é a técnica de escolha para o reparo esfincteriano. A técnica de sobreposição muscular anterior, originalmente descrita por Parks e McPartlintem, possui ampla aceitação como tratamento cirúrgico de primeira linha para incontinência fecal, a qual envolve um defeito parcial da musculatura esfincteriana, com possibilidade de identificação da porção sadia da musculatura ${ }^{33}$. As séries de reparo anterior do esfíncter incluem, em sua maioria, pacientes com defeitos devidos a causas não-obstétricas, tais como fistulotomia, com resultados imediatos variando entre $70-80 \%{ }^{36,37}$.

$\mathrm{O}$ insucesso da esfincteroplastia está geralmente associado a infecção do sítio cirúrgico, que pode ocorrer em até $24 \%$ dos casos, além da abertura da incisão após o reparo inicial, que chega a $10 \%{ }^{38}$. Dois terços dos pacientes com deiscência da área operada necessitam de nova cirurgia para tratamento de complicações, tais como incontinência ou fístula retovaginal. Os maus resultados funcionais após o reparo podem ocorrer em cerca de 50\% dos casos e se devem aos defeitos persistentes do esfíncter e no seguimento a longo prazo $^{39}$.

$\mathrm{Na}$ análise dos resultados de estudos com evolução a longo prazo da esfincteroplastia para o tratamento da incontinência fecal, observa-se queda decrescente nas taxas de sucesso da operação, de tal forma que, após 5 anos, raramente encontramos taxas superiores a $40 \%$ de sucesso $^{40,41}$.

\section{- Graciloplastia}

Essa complexa técnica descrita por Pickrell et al. ${ }^{42}$ consiste na construção de um neo-esfíncter em torno do canal anal, utilizando o músculo grácil, que resulta no estreitamento do canal anal devido ao cercamento local pelo músculo transposto. Isto resulta na obstrução a saída de fezes e em uma aparente melhora sintomática. Os resultados deste procedimento são decepcionantes, sendo que bons resultados foram observados apenas no seguimento de curto prazo. Isso se deve principalmente porque o músculo grácil é voluntário e com preponderância de fibras musculares do tipo II, que apresentam contrações forçadas por apenas um curto período de tempo, evoluindo rapidamente para fadiga, portanto, não permite uma contração sustentada ${ }^{43,44}$.

Para o refinamento dessa técnica, foi idealizada a estimulação elétrica crônica em baixa freqüência das fibras musculares esqueléticas de contração rápida (tipo 2) visando sua transformação em fibras de contração lenta sustentada (tipo 1). Essa técnica ficou conhecida como graciloplastia dinâmica, ou seja, com estimulação elétrica $^{45,46}$.

A estimulação elétrica teoricamente pode dar ao músculo grácil transposto as propriedades necessárias para funcionar como um esfíncter, porém a tecnologia é dispendiosa e depende de um eletroestimulador específico, que tem vida útil limitada. A técnica cirúrgica é elaborada e requer treinamento por longo período. Além disso, existem muitas complicações relacionadas ao método, entre as quais podemos destacar: a contração inadequada do músculo transposto, complicações infecciosas, problemas técnicos com o estimulador, rotura de tendão muscular, constipação e dor crônica ${ }^{47,48}$.

A graciloplastia dinâmica tem demonstrado taxa de sucesso inferior a $45 \%$ em dois anos, com uma maior taxa de sucesso no primeiro ano. Além disso, as taxas de complicações nestes estudos giraram em torno de 40\%, muitas delas de tratamento cirúrgico ${ }^{49,50}$.

\section{- Esfíncter artificial}

A implantação de esfíncter anal artificial (EAA) é forma mais atual de tratamento da incontinência fecal grave e representa uma alternativa ao uso da graciloplastia, sendo um procedimento menos invasivo, mas igualmente caro ${ }^{51,52}$.

A melhora da continência fecal a longo prazo com o uso de EAA, em pacientes com incontinência fecal grave, é de aproximadamente $50 \%$, dependendo da etiologia da incontinência ${ }^{51,52}$. No entanto, o implante de material sintético não biológico na região anorretal provoca risco elevado de infecção, maior do que a implantação de próteses sintéticas em outras partes do corpo $^{52}$. Esse aspecto é fundamental na determinação do sucesso dessa modalidade terapêutica. O resultado preliminar de um estudo multicêntrico americano mostrou explante da prótese decorrente de infecção local em três de 13 pacientes estudados $(23 \%)^{52}$.

\section{- Neuroestimulação sacral}

A neuroestimulação sacral apresenta grande impacto para tratamento da incontinência urinária devido a sua facilidade de uso, baixo custo e taxa de sucesso muito alta $^{10}$. Assim, esta técnica foi adaptada para o tratamento da incontinência fecal grave por Matzel et al. ${ }^{53}$ que relataram o sucesso inicial, empregando esta opção de tratamento em três pacientes. Desde então, diversos centroeuropeus e asiáticos têm empregado esta técnica para o tratamento da incontinência fecal de origem neurogênica e/ou miogênica ${ }^{54}$. Esta modalidade terapêutica consiste na colocação de eletrodos guiada por radioscopia em contato com as raízes nervosas sacrais. Estes eletrodos são acoplados a um gerador que estimula eletricamente as raízes nervosas, que, em última instância, determinam melhora da contratilidade da musculatura do esfíncter anal ${ }^{54}$.

Hull $^{54}$ publicou uma revisão na qual avaliou de 
forma crítica os resultados da neuroestimulação sacral após 15 anos de experiência mundial acumulada, concluindo ser uma opção eficaz para o tratamento da incontinência fecal, independente da etiologia. No entanto, existem resultados conflitantes na literatura. Maeda et al. ${ }^{55}$ publicaram resultados prospectivos em uma série de 176 pacientes submetidos à neuroestimulação sacral. Os autores relataram resultados sub-ótimos e eventos adversos em número significativo de pacientes no seguimento de longo prazo, destacando-se o explante do equipamento em $17,6 \%$ e a falha de funcionamento em $49,4 \%$. Além disso, há o alerta para dor pélvica crônica e o custo elevado de aplicação e manutenção ${ }^{55}$.

\section{RESULTADOS}

\section{- Transplante de órgãos pélvicos}

O transplante anorretal foi desenvolvido experimentalmente por nosso grupo ${ }^{56,58}$ e outros $^{59,62}$ como potencial tratamento da incontinência fecal grave ou colostomia definitiva. Nesses estudos os autores observaram a viabilidade da técnica cirúrgica e a recuperação funcional precoce desse enxerto após o transplante. Contudo, essa proposta não contempla o tratamento de pacientes com ID.

Os trabalhos acima descritos inspiraram o desenvolvimento do transplante inédito de órgãos perineais como proposta de tratamento das disfunções pélvicas graves acompanhadas de ID. Essa técnica se baseou em nosso estudo anatômico em cadáveres e ratos, onde observamos que os órgãos pélvicos, incluindo sua musculatura e tecido conjuntivo, são estruturas integradas e independentes que compartilham o mesmo pedículo vasculho-nervoso, representado pelas artérias e veias ilíacas internas e o nervo pudendo.

Nossa hipótese é que o procedimento lógico para tratar ID seria o transplante em bloco dos tecidos do assoalho pélvico, incluindo pele, complexo muscular pélvico, uretra, bexiga, ureter, genitália (vagina ou pênis), segmento anorretal, pedículo neurovascular e eventualmente órgãos germinativos. A seguir, descrevemos as possibilidades cirúrgicas e detalhes anatômicos para o transplante de assoalho pélvico.

\section{- Operação do doador}

Uma incisão perineal (incluindo esfíncter anal e genitália) e abdominal combinadas foram executadas. A dissecção proseguiu entre a musculatura da raiz da perna e toda musculatura perineal, que permaneceu preservada. Logo após, progredimos em torno da musculatura perineal perto do anel pélvico, incluindo esfíncter anal, reto baixo e genitalia, para promover a mobilização de todo o assoalho pélvico para dentro do abdômen (Figuras 1 e 2).

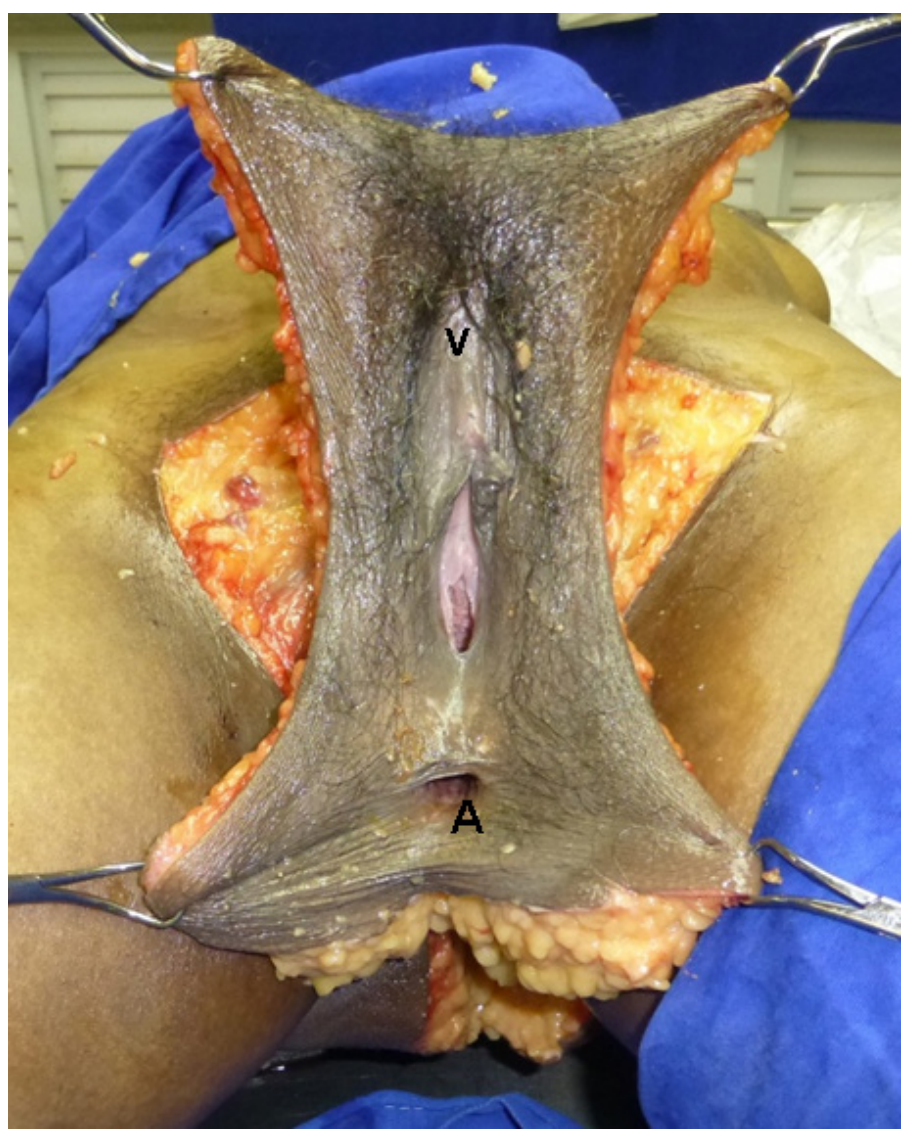

Figura 1: Aspecto da incisão e dissecção dos órgãos perineais feminino (V- Vagina, A- Ânus) 


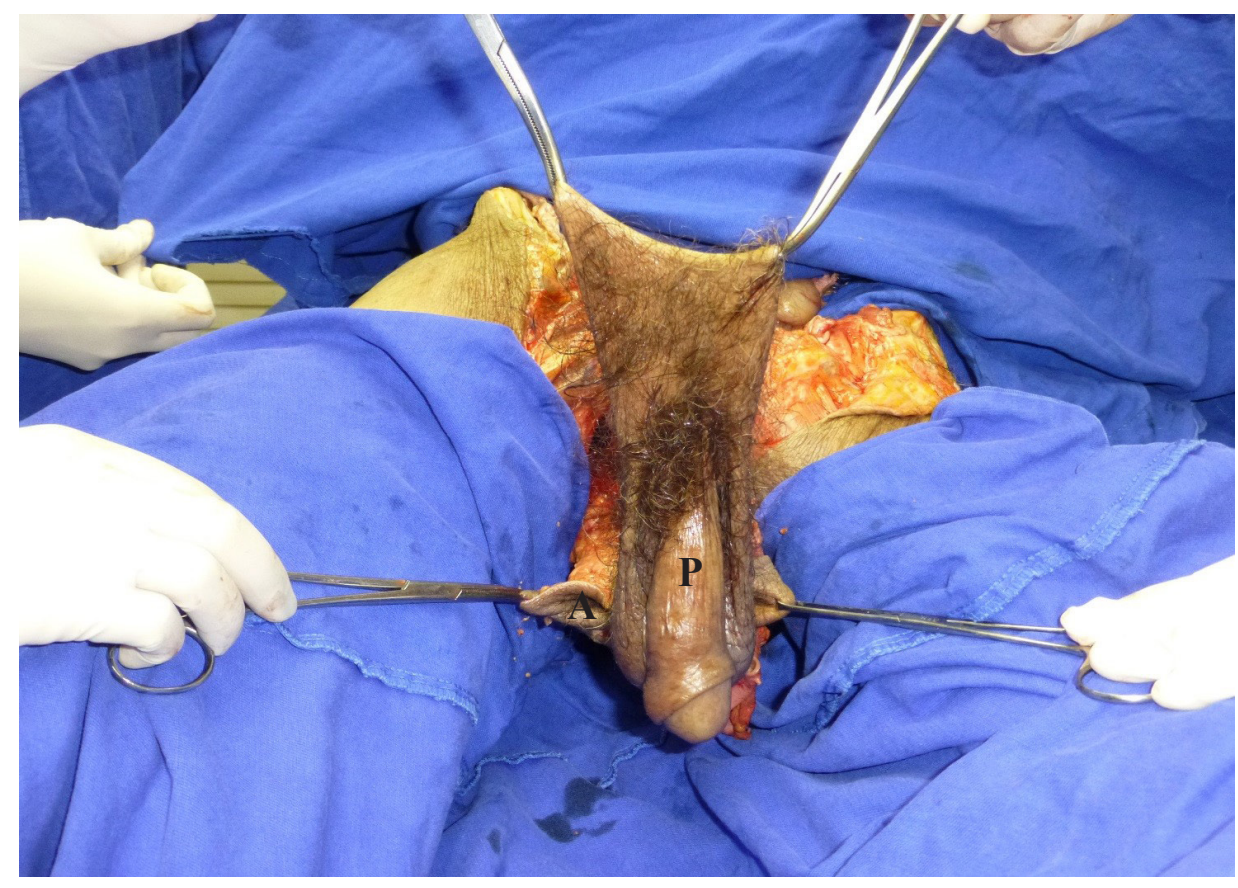

Figura 2: Aspecto da incisão e dissecção dos órgãos perineais masculino (P- Pênis, A- Ânus)
Pela incisão abdominal, os órgãos genitais, a bexiga e o reto foram mobilizados de forma que a aorta abdominal e a veia cava foram isolados até as artérias e veias renais, incluindo os vasos espermáticos. Essa dissecção se estendeu para baixo até a bifurcação ilíaca, onde os vasos ilíacos externos foram seccionados entre ligaduras, preservando os vasos ilíacos internos, incluindo os vasos retais. Nesta situação, foram identificados à direita e à esquerda os
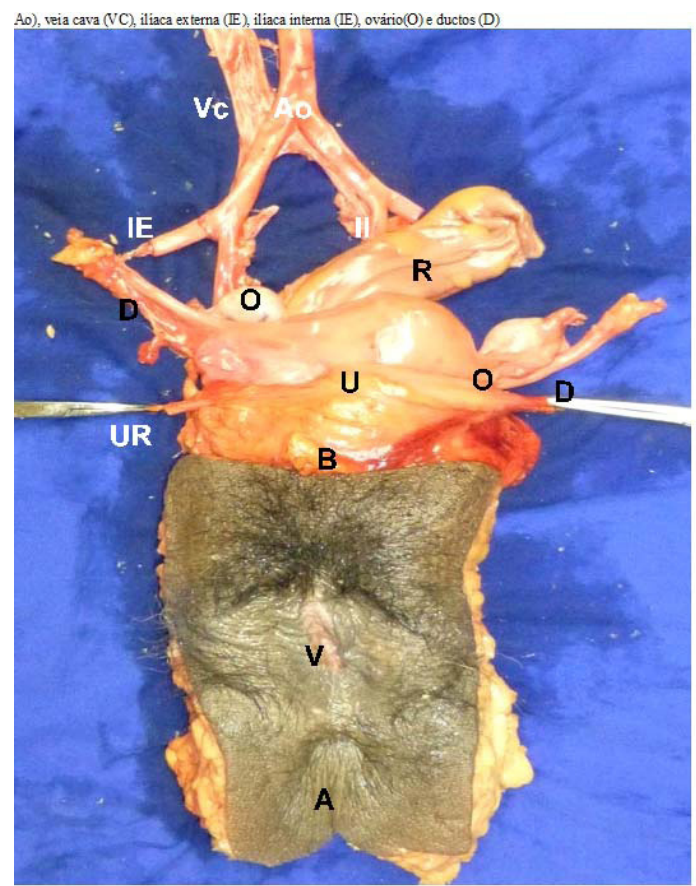

Figura 3: Enxerto pélvico contendo múltiplas estruturas: bexiga (B), ureter (UR), vagina (V), reto (R), anus (A), útero (UT), aorta (Ao), veia cava (VC), ilíaca externa (IE), ilíaca interna (IE), ovário(O) e ductos (D) nervos pudendo e seus vasos, que foram divididos próximo ao sacro. A aorta e veia cava foram seccionadas próximo aos vasos renais, o reto foi seccionado a cerca de 10 centímetros do ânus e o ureter direito e esquerdo foram seccionados próximo a bexiga. $\mathrm{O}$ enxerto contendo todos os tecidos pélvicos foi retirado (Figuras 3 e 4), colocado num recipiente contendo solução de preservação gelada e um cateter foi inserido na aorta para lavar o enxerto com solução de preservação gelada.

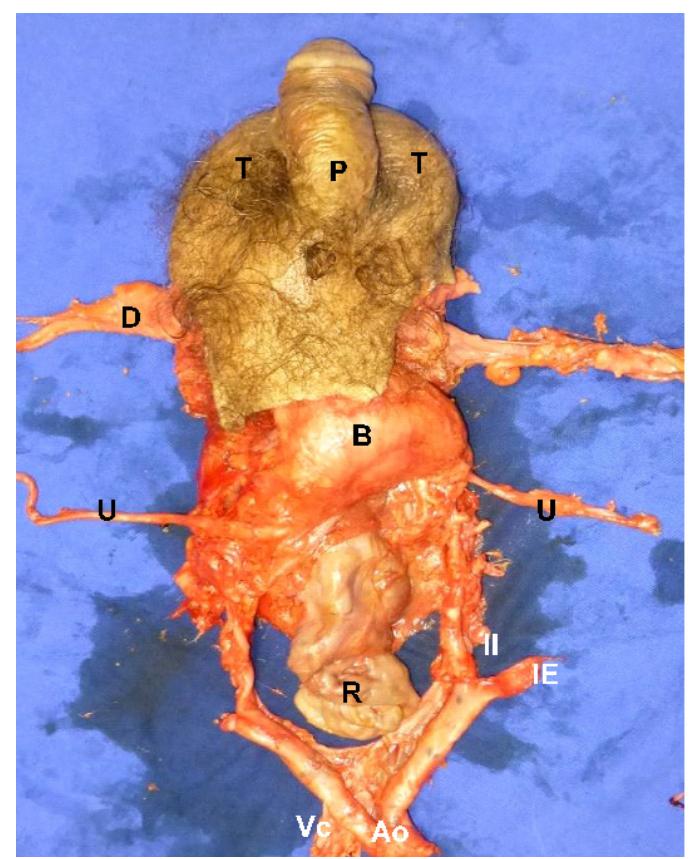

Figura 4: Enxerto pélvico contendo múltiplas estruturas: bexiga $(B)$, ureter (UR), pênis (P), reto (R), útero (UT), aorta (Ao), veia cava (VC), ilíaca externa (IE), ilíaca interna (II) e ductos (D) 


\section{- Operação do receptor}

A mesma incisão combinada perineal e abdominal feita no doador foi realizada. Um segmento de aorta e veia cava infra-renal foram isolados. $\mathrm{O}$ enxerto foi colocado no abdômen implantado por meio de anastomoses términolateral, aorta-aorta e cava-cava. O pavimento pélvico do receptor foi removido de modo semelhante ao descrito na cirurgia do doador. Foi realizada anastomose entre ureteres, nervos pudendo, seus vasos e o reto do doador e receptor. $\mathrm{O}$ enxerto foi reposicionado em sua posição original e fixado no anel pélvico, por meio de sutura de seus músculos e ligamentos. O peritôneo do assoalho pélvico, a pele do períneo e a parede abdominal foram suturados, completando a operação.

Realizamos cinco desses procedimentos em cadaveres (autotransplante) e cinco isotransplantes em ratos, em que sacrificamos os animais após 2 horas de reperfusão do enxerto. Fomos capazes de realizar todos os procedimentos. Em especial nos transplantes em ratos, observamos na cirurgia do receptor uma boa reperfusão do

\section{REFERÊNCIAS}

1. Landefeld CS, Bowers BJ, Feld AD, Hartmann KE, Hoffman E, Ingber MJ, King JT Jr, McDougal WS, Nelson H, Orav EJ, Pignone M, Richardson LH, Rohrbaugh RM, Siebens HC, Trock BJ. National institutes of health state-of-thescience conference statement: prevention of fecal and urinary incontinence in adults. Ann Intern Med. 2008;18:449-58. doi:10.7326/0003-4819-148-6-200803180-00210.

2. Lasserre A, Pelat C, Guéroult V, Hanslik T, Chartier-Kastler E, Blanchon T, Ciofu C, Montefiore ED, Alvarez FP, Bloch J. Urinary incontinence in French women: prevalence, risk factors and impact on quality of life. Eur Urol. 2009;56(1):17783. doi: 10.1016/j.eururo.2009.04.006.

3. Jackson SL, Weber AM, Hull TL, Mitchinson AR, Walters MD. Fecal incontinence in women with urinary incontinence and pelvic organ prolapse. Obstet Gynecol. 1997;89:423-7. doi:10.1016/S0029-7844(96)00499-1.

4. MeschiaM, Buonaguidi A, Pifarotti P, Somigliana E, Spennacchio M, Amicarelli F. Prevalence of anal incontinence in women with symptoms of urinary incontinence and genital prolapse. Obstet Gynecol. 2002;100:719-23.

5. Wu JM, Vaughan CP, Goode PS, Redden DT, Burgio KL, Richter HE, Markland AD. Prevalence and trends of symptomatic pelvic floor disorders in US Women. Obstet Gynecol. 2014;123:141-8. doi: 10.1097/AOG.0000000000000057.

6. Whitehead WE, Borrud L, Goode PS, Meikle S, Mueller ER, Tuteja A, Weidner A, Weinstein M, Ye W, Pelvic Floor Disorders Network. Fecal incontinence in US adults: epidemiology and risk factors. Gastroenterology. 2009;137:512-7. doi: 10.1053/j.gastro.2009.04.054.

7. González-Argenté FX, Jain A, Nogueras JJ, Davila GW, Weiss EG, Wexner SD. Prevalence and severity of urinary enxerto que se apresentava com boa coloração.

Evidentemente este procedimento ainda é experimental, porém nossos resultados preliminares em ratos mostram que este enxerto pode ser funcional. $\mathrm{O}$ transplante bloco de todo o assoalho pélvico causaria menos danos, porque preservaria todas as estruturas dessa área, incluindo os músculos e o sistema neuronal. Muitos pacientes com ID seriam beneficiados com esse procedimento, incluindo pacientes acometidos por traumas e deformações congênitas do assoalho pélvico.

\section{CONCLUSÃO}

A ID e disfunção que causa importante limitação pessoal e social, e apresenta tratamento com resultados muito pobres. O procedimento cirúrgico do transplante de órgãos pelvicos em humanos e ratos é viável. Essa inovação poderá resolver o triste problema da baixa qualidade de vida dos pacientes portadores de ID e é uma porta aberta para o debate ético, anatômico e cirúrgico.

incontinence and pelvic genital prolapse in females with anal incontinence or rectal prolapse. Dis Colon Rectum. 2001;44:920-6. Doi: 10.1007/BF02235476.

8. Fialkow MF, Melville JL, Lentz GM, Miller EA, Fenner DE. The functional and psychosocial impact of fecal incontinence in women with urinary incontinence. Am J Obstet Gynecol 2003;189:127-9. doi:10.1067/mob.2003.548.

9. Netten A, Rees T, Harrison G. Unit costs of health and social care 2001. Kent, UK: Personal Social Services Research Unit, University of Kent at Canterbury; 2001. Available from: http://www.pssru.ac.uk/pdf/UC2001/UnitCosts2001ALL.pdf.

10. Agency for Healthcare Research and Quality (AHRQ). Urinary incontinence in adults: clinical practice guideline. Rockville, MD: Agency for Health Care Policy and Research, US Dept. of Health and Human Services; 1992. (AHCPR Pub. No. 920038).

11. Johanson JF, Lafferty J. Epidemiology of fecal incontinence: the silent affliction. Am J Gastroenterol. 1996;91:33-6. Available from: http://goo.gl/y48v6Z.

12. Womack N, Morrison J, Williams N. Prospective study of the effects of postanal repair in neurogenic faecal incontinence. Br J Surg. 1988;75:48-52. doi: 10.1002/bjs. 1800750118.

13. Schuenke M, Schulte E, Schumacher U, Ross L, Lamperti E Voll W. Thieme Atlas of anatomy. New York: Thieme Medical Publishers; 2010. p.137.

14. Dawson KL, Moore SG, Rowland JM. Age-related marrow changes in the pelvis: MR and anatomic findings. Radiology. 1992;183(1):47-51. doi: http://dx.doi.org/10.1148/ radiology.183.1.1549693.

15. Corcos J, Davis MB, Drew S, West L. O uso do biofeedback 
eletromiográfico para treinar a musculatura do chão pélvico. Eletromiografia - aplicações em fisioterapia. The Biofeedback Foundation of Europe; 1997. Disponível em: http://www.bfe. org/protocol/pro04por.htm.

16. Titi M, Jenkins JT, Urie A, Molloy RG. Prospective study of the diagnostic evaluation of faecal incontinence and leakage in male patients. Colorectal Dis. 2007;9:647-52. doi: 10.1111/j.1463-1318.2006.01196.x.

17. Lindsey I, Jones OM, Smilgin-Humphreys MM, Cunningham C, Mortensen NJ. Patterns of fecal incontinence after anal surgery. Dis Colon Rectum. 2004;47:1643-9. doi: 10.1007/ s10350-004-0651-7.

18. Kim T, Chae G, Chung SS, Sands DR, Speranza JR, Weiss EG, Nogueras JJ, Wexner SD. Faecal incontinence in male patients. Colorectal Dis. 2008;10:124-30. doi: 10.1111/j.14631318.2007.01266.x.

19. Read MG, Read NW, Haynes WG, Donnelly TC, Johnson AG. A prospective study of the effect of haemorrhoidectomy on sphincter function and faecal continence. Br J Surg. 1982;69:396-8.

20. van Tets WF, Kuijpers HC. Continence disorders after anal fistulotomy. Dis Colon Rectum. 1994;37:1194-7. doi: 10.1007/BF02257781.

21. Khubchandani IT, Reed JF. Sequelae of internal sphincterotomy for chronic fissure in ano. Br J Surg. 1989;76:431-4. doi: 10.1002/bjs. 1800760504 .

22. Diokono AC, Brown MB, Brock BM, Herzog AR, Normolle DP. Clinical and cystometric characteristics of continente and incontinent noninstitutionalized elderly. J Urol 1988;140:56771.

23. Habib NA, Luck RJ: Results of transurethral resection of the benign prostate. Br J Surg 1983;70:218-21.

24. Sung VW, Washington B, Raker CA. Costs of ambulatory care related to female pelvic floor disorders in the United States. AJOG. 2010;202(5):483.e1-4. doi: 10.1016/j. ajog.2010.01.015.

25. Foley AL, Loharuka S, Barrett JA, Mathews R, Williams K, McGrother CW, Roe BH. Association between the Geriatric Giants of urinary incontinence and falls in older people using data from the Leicestershire MRC Incontinence Study. Age Ageing. 2012;41:35-40. doi: 10.1093/ageing/afr125.

26. Stewart WF, Van Rooyen JB, Cundiff GW, Abrams P, Herzog AR, Corey R, Hunt TL, Wein AJ. Prevalence and burden of overactive bladder in the United States. World J Urol. 2003;20:327-36. doi 10.1007/s00345-002-0301-4.

27. Komesu YM, Schrader RM, Rogers RG, Ketai LH. Urgency urinary incontinence in women 50 years or older: incidence, remission, and predictors of change. Female Pelvic Med Reconstr Surg. 2011;17:17-23. doi: 10.1097/ SPV.0b013e31820446e6.

28. Hall SA, Cinar A, Link CL, Kopp ZS, Roehrborn CG, Kaplan SA, Rosen RC. Do urological symptoms cluster among women? Results from the Boston Area Community Health
Survey. BJU Int. 2008;101:1257-66. doi: 10.1111/j.1464410X.2008.07557.x.

29. Perry S, McGrother CW, Turner K, Leicestershire MRC Incontinence Study Group. An investigation of the relationship between anxiety and depression and urge incontinence in women: development of a psychological model. Br J Health Psychol. 2006;11:463-82. doi: 10.1348/135910705X60742.

30. Coyne KS, Wein A, Nicholson S, Kvasz M, Chen CI, Milsom I. Comorbidities and personal burden of urgency urinary incontinence: a systematic review. Int J Clin Pract. 2013;67(10):1015-33. doi: 10.1111/ijcp.12164.

31. Eason E, Labrecque M, Marcoux S, Mondor M. Anal incontinence after childbirth. CMAJ. 2002;166:326-30. Available from: http://www.cmaj.ca/content/166/3/326.full. pdf + html.

32. Hallgren T, Fasth S, Delbro DS, Nordgren S, Oresland T, Hulten L. Loperamide improves anal sphincter function and continence after restorative proctocolectomy. Dig Dis Sci. 1994;39:2612-8. doi: 10.1007/BF02087698.

33. Christiansen J, Roed-Petersen K. Clinical assessment of the anal continence plug. Dis Colon Rectum. 1993;36:740-2. doi: 10.1007/BF02048363.

34. Norton C, Hosker G, Brazzelli M. Biofeedback and/or sphincter exercises for the treatment of fecal incontinence in adults. Cochrane Database Syst Rev. 2000;2:CD 002111. doi: 10.1002/14651858.CD002111.

35. Parks AG, McPartlin JF. Later repair of injuries of the anal sphincter. Proc R Soc Med. 1971;64:1187-9. Available from: http://www.ncbi.nlm.nih.gov/pmc/articles/PMC1813153/pdf/ procrsmed00275-0029.pdf.

36. Oliveira L, Pfeifer J, Wexner SD. Physiological and clinical outcome of anterior sphincteroplasty. Br J Surg. 1996;83:5025.

37. Barisic GI, Krivokapic ZV, Markovic VA, Popovic MA. Outcome of overlapping anal sphincter repair after 3 months and after a mean of 80 months. Int J Colorectal Dis. 2006;21(1):52-6. doi: 10.1007/s00384-004-0737-7.

38. Jacobs PP, Scheuer M, Kuijpers JH, Vingerhoets MH. Obstetric fecal incontinence. Role of pelvic floor denervation and results of delayed sphincter repair. Dis Colon Rectum. 1990;33:494-7. doi: 10.1007/BF02052145.

39. Cook TA. McMortensen NJ. Management of faecal incontinence following obstetric injury. Br J Surg. 1998,85:293-9. doi: 10.1046/j.1365-2168.1998.00693.x.

40. Rothbarth J, Bemelman WA, Meijerink WJHJ, BuyzeWesterweel ME, van Dijk JG, Delemarre JBVM. Long-term results of anterior anal sphincter repair for fecal incontinence due to obstetric injury. Dig Surg. 2000;17:390-4. doi: $10.1159 / 000018883$.

41. Bravo Gutierrez A, Madoff RD, Lowry AC, Parker SC, Buie WD, Baxter NN. Long-term results of anterior sphincteroplasty. Dis Colon Rectum. 2004;47:727-32. doi: 10.1007/s10350-003-0114-6. 
42. Pickrell KL, Broadbent TR, Masters FW, Metzger JT. Construction of a rectal sphincter and restoration of anal continence by transplanting the gracilis muscle: a report of four cases in children. Ann Surg. 1952;135:853-62. Available from: http://www.ncbi.nlm.nih.gov/pmc/articles/ PMC1802208/pdf/annsurg01429-0103.pdf.

43. Fleshner PR, Roberts PL. Encirclement procedures for fecal incontinence. Perspect Colon Rectal Surg. 1991;4:280-7.

44. Kumar D, Hutchinson R, Grant E. Bilateral gracilis neosphincter construction for treatment of faecal incontinence. Br J Surg. 1995;82:1645-7.

45. Konsten J, Baeten CG, Spaans F, Havenith MG, Soeters PB. Follow-up of anal dynamic graciloplasty for fecal continence. World J Surg. 1993;17:404-9.

46. George BD, Williams NS, Patel J, Swash M, Watkins ES. Physiological and histochemical adaptation of the electrically stimulated graciles muscle to neoanal sphincter function. $\mathrm{Br} \mathrm{J}$ Surg. 1993;80:1342-6. doi: 10.1002/bjs.1800801042.

47. Wexner SD, Gonzalez-Padron A, Ruis J, Teoh TA, Cheong DM, Nogueras JJ, Billotti VL, Weiss EG, Moon HK. Stimulated gracilis neosphincter operation. Initial experience, pitfalls, and complications. Dis Colon Rectum. 1996;39:95764. doi: 10.1007/BF02054681.

48. Geerdes BP, Heineman E, Konsten J, Soeters PB, Baeten CGMI. Dynamic graciloplasty. Complications and management. Dis Colon Rectum. 1996;39:912-7.

49. Penninckx F. On behalf of the Belgian Section of Colorectal Surgery. Belgian experience with dynamic graciloplasty for faecal incontinence. Br J Surg. 2004;91:872-8. doi: 10.1002/ bjs. 4568 .

50. Matzel KE, Madoff RD, La Fontaine LJ, Baeten CG, Buie WD, Christiansen J, Wexner S; Dynamic Graciloplasty Therapy Study Group. Complications of dynamic graciloplasty: incidence, management, and impact on outcome. Dis Colon Rectum. 2001;44:1427-35. doi: 10.1007/BF02234593.

51. Christiansen J, Sparsø B. Treatment of anal incontinence by an implantable prosthetic anal sphincter. Ann Surg. 1992;215:383-6. Available from: http://www.ncbi.nlm.nih. gov/pmc/articles/PMC1242456/pdf/annsurg00086-0107.pdf.

52. Spencer M, Wong W, Congilosi S, et al. Artificial anal sphincter: preliminary results of a multicenter prospective trial. Dis Colon Rectum. 1998;41:A15.

53. Matzel KE, Stadelmaier U, Hohenfellner M, Gall FP. Electrical stimulation of sacral spinal nerves for treatment of faecal incontinence. Lancet. 1995;346:1124-7. Available from: http://goo.gl/9Te2bk.

54. Hull T. Sacral neuromodulation stimulation in fecal incontinence. Int Urogynecol J. 2010;21:1565-8. doi: 10.1007/s00192-010-1283-1.

55. Maeda Y, Lundby L, Buntzen S, Laurberg S. Suboptimal outcome following sacral nerve stimulation for faecal incontinence. Br J Surg. 2011;98:140-7. doi: 10.1002/ bjs. 7302 .

56. Galvao FH, Seid VE, Waisberg DR, Cruz RJ Jr, Hirano H, Catanozi S, Chaib E, D'Albuquerque LA. An innovative model of autologous anorectal transplantation with pudendal nerve reconstruction. Clinics (Sao Paulo). 2012;67(8):971-2. doi: 10.6061/clinics/2012(08)20.

57. Galvão FH, Waisberg DR, Vianna RM, Galvão RC, Seid VE, Andraus W, Chaib E, D'Albuquerque LA. Intestinal transplantation including anorectal segment in the rat. Microsurgery. 2012;32(1):77-9. doi: 10.1002/micr.20958.

58. Galvão FH, Seid VE, Nunes dos Santos RM, Kitamura M, de Castro Galvão R, Ambar Pinto R, Miyashiro Nunes dos Santos R. Anorectal transplantation. Tech Coloproctol. 2009;13(1):55-9. doi: 10.1007/s10151-009-0459-5.

59. Araki J, Nishizawa Y, Sato T, Naito M, Akita K, Tashiro $\mathrm{K}$, Iida T, Koshima I. Anorectal transplantation in human cadavers: mock anorectal allotransplantation. PLoS One. 2013;8(7):e68977. doi: 10.1371/journal.pone.0068977.

60. Araki J, Nishizawa Y, Nakamura T, Sato T, Naito M, Fujii S, Mihara M, Koshima I. The development of a canine anorectal autotransplantation model based on blood supply: a preliminary case report. PLoS One. 2012;7(9):e44310. doi: 10.1371/journal.pone.0044310.

61. Araki J, Mihara M, Narushima M, Iida T, Sato T, Koshima I. Vascularized anal autotransplantation model in rats: preliminary report. Transplant Proc. 2011;43(9):3552-6. doi: 10.1016/j.transproceed.2011.08.042.

62. O’Bichere A, Shurey S, Sibbons P, Green C, Phillips RK. Experimental model of anorectal transplantation. Br J Surg. 2000;87(11):1534-9. doi: 10.1046/j.1365-2168.2000.01557.x. 